\title{
On well-covered, vertex decomposable and Cohen-Macaulay graphs
}

\author{
Iván D. Castrillón* \\ Departamento de Matemáticas \\ Centro de Investigación y de Estudios Avanzados del IPN \\ Ciudad de México, México \\ idcastrillon@math.cinvestav.mx \\ Roberto Cruz \\ Instituto de Matemáticas \\ Universidad de Antioquia \\ Medellín, Colombia \\ roberto.cruz@udea.edu.co

\section{Enrique Reyes} \\ Departamento de Matemáticas \\ Centro de Investigación y de Estudios Avanzados del IPN \\ Ciudad de México, México \\ ereyes@math. cinvestav.mx
}

Submitted: Jan 17, 2016; Accepted: May 10, 2016; Published: May 27, 2016

Mathematics Subject Classifications: 13F55, 05E40, 05E45, 05C75

\begin{abstract}
Let $G=(V, E)$ be a graph. If $G$ is a König graph or if $G$ is a graph without 3 -cycles and 5 -cycles, we prove that the following conditions are equivalent: $\Delta_{G}$ is pure shellable, $R / I_{\Delta}$ is Cohen-Macaulay, $G$ is an unmixed vertex decomposable graph and $G$ is well-covered with a perfect matching of König type $e_{1}, \ldots, e_{g}$ without 4-cycles with two $e_{i}$ 's. Furthermore, we study vertex decomposable and shellable (non-pure) properties in graphs without 3-cycles and 5-cycles. Finally, we give some properties and relations between critical, extendable and shedding vertices.
\end{abstract}

Keywords: Cohen-Macaulay, shellable, well-covered, unmixed, vertex decomposable, König, girth

\section{Introduction}

Let $G$ be a simple graph (without loops and multiplies edges) whose vertex set is $V(G)=$ $\left\{x_{1}, \ldots, x_{n}\right\}$ and edge set $E(G)$. Let $R=k\left[x_{1}, \ldots, x_{n}\right]$ be a polynomial ring over a field $k$. The edge ideal of $G$, denoted by $I(G)$, is the ideal of $R$ generated by all monomials $x_{i} x_{j}$ such that $\left\{x_{i}, x_{j}\right\} \in E(G)$. $G$ is a Cohen-Macaulay graph if $R / I(G)$ is a Cohen-Macaulay

\footnotetext{
*Partially supported by CONACYT and ABACUS-CINVESTAV.
} 
ring (see [3], [20]). A subset $F$ of $V(G)$ is a stable set or independent set if $e \nsubseteq F$ for each $e \in E(G)$. The cardinality of the maximum stable set is denoted by $\beta(G)$. $G$ is called well-covered if every maximal stable set has the same cardinality. On the other hand, a subset $D$ of $V(G)$ is a vertex cover of $G$ if $D \cap e \neq \varnothing$ for every $e \in E(G)$. The number of vertices in a minimum vertex cover of $G$ is called the covering number of $G$ and it is denoted by $\tau(G)$. This number coincide with ht $(I(G))$, the height of $I(G)$. If the minimal vertex covers have the same cardinality, then $G$ is called an unmixed graph. Notice that, $D$ is a vertex cover if and only if $V(G) \backslash D$ is a stable set. Hence, $\tau(G)=n-\beta(G)$ and $G$ is well-covered if and only if $G$ is unmixed. The Stanley-Reisner complex of $I(G)$, denoted by $\Delta_{G}$, is the simplicial complex whose faces are the stable sets of $G$. Recall that a simplicial complex $\Delta$ is called pure if every facet has the same number of elements. Thus, $\Delta_{G}$ is pure if and only if $G$ is well-covered.

Some properties of $G, \Delta_{G}$ and $I(G)$ allow an interaction between Commutative Algebra and Combinatorial Theory. Examples of these properties are: Cohen-Macaulayness, shellability, vertex decomposability and well-coveredness. These properties have been studied in ([3], [4], [6], [7], [11], [12], [13], [16], [17], [18], [20], [22]). In general, we have the following implications (see [3], [16], [20], [22])

$$
\underset{\text { vertex decomposable }}{\text { Unmixed }} \Rightarrow \begin{gathered}
\text { Pure } \\
\text { shellable }
\end{gathered} \Rightarrow \text { Cohen-Macaulay } \Rightarrow \text { Well-covered }
$$

The equivalence between the Cohen-Macaulay property and the unmixed vertex decomposable property has been studied for some families of graphs: bipartite graphs (in [7] and [11]); very well-covered graphs (in [5] and [13]); graphs with girth at least 5, blockcactus (in [12]); and graphs without 4-cycles and 5-cycles (in [2]). For this paper, a cycle $C=\left(z_{1}, z_{2}, \ldots, z_{n}\right)$ can have chords (edges between non-consecutive vertices in $C$ ) in $G$. A cycle without chords is called an induced cycle.

If a bipartite graph is well-covered, pure shellable or Cohen-Macaulay, then it is König and has a perfect matching. The perfect matching is important because it allowed Hibi and Herzog to characterize Cohen-Macaulay bipartite graph (see [11]). Similarly, the existence of a perfect matching allows one to find a classification of well-covered bipartite graphs (see [15] and [19]). However, a 3-cycle and a 5-cycle are Cohen-Macaulay graphs, but they does not have a perfect matching. This is the motivation for the study of Cohen-Macaulay graphs without 3-cycles and 5-cycles. In particular, we are interested in knowing if these graphs have a perfect matching. In this paper we prove that it is affirmative.

The paper is organized as follow: in section 2 we give some properties and relations between critical, shedding and extendable vertices that we will use in the following sections. In section 3 we prove some results about well-covered graphs. In section 4 we prove the equivalences of unmixed vertex decomposable and Cohen-Macaulay properties for König graphs and graphs without 3-cycles and 5-cycles. We prove that theses properties are equivalent to the following condition: $G$ is an unmixed König graph with a perfect matching $e_{1}, \ldots, e_{g}$ without 4 -cycles with two $e_{i}$ 's. This result extends the criterion of 
Herzog-Hibi for Cohen-Macaulay bipartite graphs, given in [11]. In [17] Van Tuyl proved that the vertex decomposable property, the shellable (non-pure) property and the sequentially Cohen-Macaulay property are equivalent in bipartite graphs. Furthermore, in [18] Van Tuyl and Villarreal give a criterion that characterize shellable bipartite graphs. These results and results obtained in section 4, motivate us to study the vertex decomposable property and the shellable (non-pure) property for graphs without 3-cycles and 5-cycles. In section 5, we prove that the neighborhood of a 2-connected block of $G$ has a free vertex, if $G$ is a bipartite shellable graph or if $G$ is a vertex decomposable graph without 3-cycles and 5-cycles. Also, we prove that the criterion of Van Tuyl-Villarreal can be extended to vertex decomposable graphs without 3-cycles and 5-cycles and shellable graphs with girth at least 11 . The equivalence between the shellable property and the vertex decomposable property for graphs without 3-cycles and 5-cycles is an open problem.

\section{Critical, extendable and shedding vertices.}

Let $X$ be a subset of $V(G)$. The subgraph induced by $X$ in $G$, denoted by $G[X]$ is the graph with vertex set $X$ and whose edge set is $\{\{x, y\} \in E(G) \mid x, y \in X\}$. Furthermore, let $G \backslash X$ denote the induced subgraph $G[V(G) \backslash X]$. Now, if $v \in V(G)$, then the set of neighbors of $v$ (in $G$ ) is denoted by $N_{G}(v)$ and its closed neighborhood is $N_{G}[v]=N_{G}(v) \cup\{v\}$. The degree of $v$ in $G$ is $\operatorname{deg}_{G}(v)=\left|N_{G}(v)\right|$.

Definition 1. $G$ is vertex decomposable if $G$ is a totally disconnected graph or there is a vertex $v$ such that

(a) $G \backslash v$ and $G \backslash N_{G}[v]$ are both vertex decomposable, and

(b) each stable set in $G \backslash N_{G}[v]$ is not a maximal stable set in $G \backslash v$.

A shedding vertex of $G$ is any vertex which satisfies the condition (b). Equivalently, $v$ is a shedding vertex if for every stable set $S$ contained in $G \backslash N_{G}[v]$, there is some $x \in N_{G}(v)$ such that $S \cup\{x\}$ is stable.

Lemma 2. If $x$ is a vertex of $G$, then $x$ is a shedding vertex if and only if $\left|N_{G}(x)\right|$ $N_{G}(S) \mid \geqslant 1$ for every stable set $S$ of $G \backslash N_{G}[x]$.

Proof. $\Rightarrow)$ We take a stable set $S$ of $G \backslash N_{G}[x]$. Since $x$ is a shedding vertex, then there is a vertex $z \in N_{G}(x)$ such that $S \cup\{z\}$ is a stable set of $G \backslash x$. Thus, $z \notin N_{G}[S]$. Therefore, $\left|N_{G}(x) \backslash N_{G}(S)\right| \geqslant 1$.

$\Leftarrow)$ We take a stable set $S$ of $G \backslash N_{G}[x]$. Thus, there exists a vertex $z \in N_{G}(x) \backslash N_{G}(S)$. Since $z \in N_{G}(x)$, we have that $z \notin S$. Furthermore, $z \notin N_{G}(S)$, then $S \cup\{z\}$ is a stable set of $G \backslash x$. Consequently, $S$ is not a maximal stable set of $G \backslash x$. Therefore, $x$ is a shedding vertex.

Definition 3. Let $S$ be a stable set of $G$. If $x$ is of degree zero in $G \backslash N_{G}[S]$, then $x$ is called isolated vertex in $G \backslash N_{G}[S]$, or we say that $S$ isolates to $x$. 
By Lemma 2, we have that $x$ is not a shedding vertex if and only if there exists a stable set $S$ of $G \backslash N_{G}[x]$ such that $N_{G}(x) \subseteq N_{G}(S)$, i.e. $x$ is an isolated vertex in $G \backslash N_{G}[S]$.

Corollary 4. Let $S$ be a stable set of $G$. If $S$ isolates $x$ in $G$, then $x$ is not a shedding vertex in $G \backslash N_{G}[y]$ for all $y \in S$.

Proof. Since $S$ isolates $x$, then $\operatorname{deg}_{G \backslash N_{G}[S]}(x)=0$ and in particular $x \in V\left(G \backslash N_{G}[S]\right)$. Thus, $N_{G}(x) \subseteq N_{G}[S] \backslash S$. Hence, if $y \in S$ and $G^{\prime}=G \backslash N_{G}[y]$, then $x \in V\left(G^{\prime}\right)$. Furthermore, since $S \cap N_{G}[x]=\varnothing$, then $S^{\prime}=S \backslash y$ is a stable set in $G^{\prime} \backslash N_{G^{\prime}}[x]$. Now, since $S$ isolates $x$, if $a \in N_{G^{\prime}}(x)$, then there exists $s \in S$ such that $\{a, s\} \in E(G)$. But $a \in N_{G^{\prime}}(x)$, then $a \notin N_{G}[y]$, consequently $s \in S^{\prime}$ and $\{a, s\} \in E\left(G^{\prime}\right)$. This implies $\left|N_{G^{\prime}}(x) \backslash N_{G^{\prime}}\left(S^{\prime}\right)\right|=0$. Therefore, by Lemma $2, x$ is not a shedding vertex in $G^{\prime}$.

Theorem 5. If $x$ is a shedding vertex of $G$, then one of the following conditions hold:

(a) There is $y \in N_{G}(x)$ such that $N_{G}[y] \subseteq N_{G}[x]$.

(b) $x$ is in a 5-cycle with at most one chord.

Proof. We take $N_{G}(x)=\left\{y_{1}, y_{2}, \ldots, y_{k}\right\}$. If $G$ does not satisfy (a), then there is

$$
\left\{z_{1}, \ldots, z_{k}\right\} \subseteq V(G) \backslash N_{G}[x]
$$

such that $\left\{y_{i}, z_{i}\right\} \in E(G)$ for $i \in\{1, \ldots, k\}$. We denote by $L=\left\{z_{1}, \ldots, z_{q}\right\}=\left\{z_{1}, \ldots, z_{k}\right\}$ and suppose that $z_{i} \neq z_{j}$ for $1 \leqslant i<j \leqslant q$. By Lemma 2, if $L$ is a stable set of $G$, then $\left|N_{G}(x) \backslash N_{G}(L)\right| \geqslant 1$. But $N_{G}(x)=\left\{y_{1}, \ldots, y_{k}\right\} \subseteq N_{G}(L)$, then $L$ is not a stable set. Hence, $q \geqslant 2$ and there exist $z_{i_{1}}, z_{i_{2}} \in L$ such that $\left\{z_{i_{1}}, z_{i_{2}}\right\} \in E(G)$. Thus, there exist $y_{j_{1}}$ and $y_{j_{2}}$ such that $y_{j_{1}} \neq y_{j_{2}}$ and $\left\{y_{j_{1}}, z_{i_{1}}\right\},\left\{y_{j_{2}}, z_{i_{2}}\right\} \in E(G)$. Furthermore, $\left\{z_{i}, y_{j_{2}}\right\},\left\{z_{i_{2}}, y_{j_{1}}\right\},\left\{z_{i_{1}}, x\right\},\left\{z_{i_{2}}, x\right\} \notin E(G)$. Therefore, $\left(x, y_{j_{1}}, z_{i_{1}}, z_{i_{2}}, y_{j_{2}}\right)$ is a 5 -cycle of $G$ with at most one chord.

Definition 6. A vertex $v$ is called simplicial if the induced subgraph $G\left[N_{G}(v)\right]$ is a complete graph (or clique). Equivalently, a simplicial vertex is a vertex that appears in exactly one clique.

Remark 7. If $v, w \in V(G)$ such that $N_{G}[v] \subseteq N_{G}[w]$, then $w$ is a shedding vertex of $\mathrm{G}$ (see Lemma 6 in [22]). In particular, if $v$ is a simplicial vertex, then any $w \in N_{G}(v)$ is a shedding vertex (see Corollary 7 in [22]).

Corollary 8. Let $G$ be graph without 4-cycles. If $x$ is a shedding vertex of $G$, then $x$ is in a 5-cycle or there exists a simplicial vertex $z$ such that $\{x, z\} \in E(G)$ with $\left|N_{G}[z]\right| \leqslant 3$.

Proof. By Theorem 5, if $x$ is not in a 5-cycle, then there is $z \in N_{G}(x)$ such that $N_{G}[z] \subseteq$ $N_{G}[x]$. If $\operatorname{deg}_{G}(z)=1$, then $z$ is a simplicial vertex. If $\operatorname{deg}_{G}(z)=2$, then $N_{G}(z)=\{x, w\}$. Consequently, $(z, x, w)$ is a 3 -cycle since $N_{G}[z] \subseteq N_{G}[x]$. Thus, $z$ is a simplicial vertex. Now, if $\operatorname{deg}_{G}(z) \geqslant 3$, then there are $w_{1}, w_{2} \in N_{G}(z) \backslash x$. Since $N_{G}[z] \subseteq N_{G}[x]$, we have that $\left(w_{1}, z, w_{2}, x\right)$ is a 4 -cycle of $G$. This is a contradiction. Therefore, $\left|N_{G}[z]\right| \leqslant 3$ and $z$ is a simplicial vertex. 
Remark 9. If $G$ is a 5-cycle with $V(G)=\left\{x_{1}, x_{2}, x_{3}, x_{4}, x_{5}\right\}$, then each $x_{i}$ is a shedding vertex.

Proof. We can assume that $i=1$, then $\left\{x_{3}\right\}$ and $\left\{x_{4}\right\}$ are the stable sets in $G \backslash N_{G}\left[x_{1}\right]$. Furthermore, $\left\{x_{3}, x_{5}\right\}$ and $\left\{x_{2}, x_{4}\right\}$ are stable sets in $G \backslash x_{1}$. Hence, each stable set of $G \backslash N_{G}\left[x_{1}\right]$ is not a maximal stable set in $G \backslash x_{1}$. Therefore, $x_{1}$ is a shedding vertex.

Definition 10. A vertex $v$ of $G$ is critical if $\tau(G \backslash v)<\tau(G)$. Furthermore, $G$ is called a vertex critical graph if each vertex of $G$ is critical.

Lemma 11. If $\tau(G \backslash v)<\tau(G)$, then $\tau(G)=\tau(G \backslash v)+1$. Moreover, $v$ is a critical vertex if and only if $\beta(G)=\beta(G \backslash v)$.

Proof. If $t$ is a minimal vertex cover such that $|t|=\tau(G \backslash v)$, then $t \cup\{v\}$ is a vertex cover of $G$. Thus, $\tau(G) \leqslant|t \cup\{v\}|=\tau(G \backslash v)+1$. Consequently, if $\tau(G)>\tau(G \backslash v)$, then $\tau(G)=\tau(G \backslash v)+1$.

Now, we have that $\tau(G)+\beta(G)=|V(G)|=|V(G \backslash v)|+1=\tau(G \backslash v)+\beta(G \backslash v)+1$. Hence, $\beta(G)=\beta(G \backslash v)$ if and only if $\tau(G)=\tau(G \backslash v)+1$. Therefore, $v$ is a critical vertex if and only if $\beta(G)=\beta(G \backslash v)$.

Definition 12. A vertex $v$ of $G$ is called an extendable vertex if $G$ and $G \backslash v$ are wellcovered graphs with $\beta(G)=\beta(G \backslash v)$.

Note that if $v$ is an extendable vertex, then every maximal stable set $S$ of $G \backslash v$ contains a vertex of $N_{G}(v)$.

Corollary 13. Let $G$ be an unmixed graph and $x \in V(G)$. The following conditions are equivalent:

(a) $x$ is an extendable vertex.

(b) $\left|N_{G}(x) \backslash N_{G}(S)\right| \geqslant 1$ for every stable set $S$ of $G \backslash N_{G}[x]$.

(c) $x$ is a shedding vertex.

(d) $x$ is a critical vertex and $G \backslash x$ is unmixed.

Proof. $(a) \Leftrightarrow(b)([8]$, Lemma 2).

$(b) \Leftrightarrow(c)$ By Lemma 2.

$(a) \Leftrightarrow(d)$ Since $G$ is unmixed, then by Lemma $11, x$ is extendable if and only if $x$ is a critical vertex and $G \backslash x$ is unmixed. 


\section{König and well-covered graphs}

In this paper we denoted by $Z_{G}$ the set of the isolated vertices of $G$, that is,

$$
Z_{G}=\left\{x \in V(G) \mid \operatorname{deg}_{G}(x)=0\right\} .
$$

Definition 14. $G$ is a König graph if $\tau(G)=\nu(G)$ where $\nu(G)$ is the maximum number of pairwise disjoint edges. A perfect matching of König type of $G$ is a collection $e_{1}, \ldots, e_{g}$ of pairwise disjoint edges whose union is $V(G)$ and $g=\tau(G)$.

Proposition 15. Let $G$ be a König graph and $G^{\prime}=G \backslash Z_{G}$. Then the following are equivalent:

(a) $G$ is unmixed.

(b) $G^{\prime}$ is unmixed.

(c) If $V\left(G^{\prime}\right) \neq \varnothing$, then $G^{\prime}$ has a perfect matching $e_{1}, \ldots, e_{g}$ of König type such that for any two edges $f_{1} \neq f_{2}$ and for two distinct vertices $x \in f_{1}, y \in f_{2}$ contained in some $e_{i}$, one has that $\left(f_{1} \backslash x\right) \cup\left(f_{2} \backslash y\right)$ is an edge.

Proof. (a) $\Leftrightarrow$ (b) Since $V(G) \backslash V\left(G^{\prime}\right)=Z_{G}$, then $C$ is a vertex cover of $G$ if and only if $C$ is a vertex cover of $G^{\prime}$. Therefore, $G$ is unmixed if and only if $G^{\prime}$ is unmixed.

$(\mathrm{b}) \Leftrightarrow(\mathrm{c})$ By $([14]$, Lemma 2.3 and Proposition 2.9).

Definition 16. A graph $G$ is called very well-covered if it is well-covered without isolated vertices and $|V(G)|=2 \mathrm{ht}(I(G))$.

Lemma 17. $G$ is an unmixed König graph if and only if $G$ is totally disconnected or $G^{\prime}=G \backslash Z_{G}$ is very well-covered.

Proof. $\Rightarrow)$ If $G$ is not totally disconnected, then from Proposition $15, G^{\prime}$ has a perfect matching $e_{1}, \ldots, e_{g}$ of König type. Hence, $\left|V\left(G^{\prime}\right)\right|=2 g=2 \tau\left(G^{\prime}\right)=2 \mathrm{ht}\left(I\left(G^{\prime}\right)\right)$. Furthermore, $G^{\prime}$ is unmixed, therefore $G^{\prime}$ is very well-covered.

$\Leftarrow)$ If $G$ is totally disconnected, then $\nu(G)=0$ and $\tau(G)=0$. Hence, $G$ is an unmixed König graph. Now, if $G$ is not totally disconnected, then $G^{\prime}$ is very well-covered. Consequently, by ([10], Corollary 3.7) $G^{\prime}$ has a perfect matching. Thus, $\nu\left(G^{\prime}\right)=\left|V\left(G^{\prime}\right)\right| / 2=$ $\operatorname{ht}\left(G^{\prime}\right)=\tau\left(G^{\prime}\right)$. Hence, $G^{\prime}$ is König. Furthermore, $\nu(G)=\nu\left(G^{\prime}\right)$ and $\tau(G)=\tau\left(G^{\prime}\right)$, then $G$ is König. Finally, since $G^{\prime}$ is unmixed, by Proposition 15, $G$ is also unmixed.

Definition 18. A subgraph $H$ of $G$ is called a c-minor (of $G$ ) if there exists a stable set $S$ of $G$, such that $H=G \backslash N_{G}[S]$.

Remark 19. Each connected component of a graph $G$ is a c-minor of $G$.

Remark 20. The unmixed property is closed under c-minors. That is, each c-minor of $G$ has the same property (see [20]). 
Definition 21. A vertex of degree one is called leaf or free vertex. Furthermore, an edge which is incident with a leaf is called pendant.

Lemma 22. If $G$ is an unmixed graph and $x \in V(G)$, then $N_{G}(x)$ does not contain two free vertices.

Proof. We suppose that there exists $x \in V(G)$ such that $y_{1}, \ldots, y_{s}$ are free vertices in $N_{G}(x)$. Hence, $G_{1}=G \backslash N_{G}\left[y_{1}, \ldots, y_{s}\right]=G \backslash\left\{x, y_{1}, \ldots, y_{s}\right\}$ is unmixed. Now, we take a maximal stable set $S$ of $G_{1}$. Thus, $|S|=\beta\left(G_{1}\right)$ since $G_{1}$ is unmixed. Consequently, $S_{1}=S \cup\left\{y_{1}, \ldots, y_{s}\right\}$ is a stable set in $G$. We take $S_{2}$ a maximal stable in $G$ such that $x \in S_{2}$. Since $G$ is unmixed, we have that $\left|S_{2}\right| \geqslant\left|S_{1}\right|=|S|+s$. Furthermore, $S_{2} \backslash x$ is a stable set in $G_{1}$, then $\left|S_{2}\right| \leqslant \beta\left(G_{1}\right)+1$. This implies $\beta\left(G_{1}\right)+1 \geqslant|S|+s$. But, $|S|=\beta\left(G_{1}\right)$, therefore, $s \leqslant 1$.

Definition 23. If $v, w \in V(G)$, then the distance $\mathrm{d}(u, v)$ between $u$ and $v$ in $G$ is the length of the shortest path joining them, otherwise $d(u, v)=\infty$. Now, if $H \subseteq G$, then the distance from a vertex $v$ to $H$ is $d(v, H)=\min \{d(v, u) \mid u \in V(H)\}$. Furthermore, if $W \subseteq V(G)$, then we define $d(v, W)=d(v, G[W])$ and $D_{i}(W)=\{v \in V(G) \mid d(v, W)=i\}$.

Proposition 24. Let $G$ be an unmixed connected graph without 3-cycles and 5-cycles. If $C$ is a 7-cycle and $H$ is a c-minor of $G$ with $C \subseteq H$ such that $C$ has three non-adjacent vertices of degree 2 in $H$, then $C$ is a c-minor of $G$.

Proof. We take a minimal c-minor $H$ of $G$ such that $C \subseteq H$ and $C$ has three non-adjacent vertices of degree 2 in $H$. We can suppose that $C=\left(x, z_{1}, w_{1}, a, b, w_{2}, z_{2}\right)$ with $\operatorname{deg}_{H}(x)=$ $\operatorname{deg}_{H}\left(w_{1}\right)=\operatorname{deg}_{H}\left(w_{2}\right)=2$. If $\left\{z_{1}, b\right\} \in E(H)$, then $\left(z_{1}, b, w_{2}, z_{2}, x\right)$ is a 5-cycle of $G$. Thus, $\left\{z_{1}, b\right\} \notin E(H)$, similarly $\left\{z_{2}, a\right\} \notin E(H)$. Furthermore, since $G$ does not have 3 -cycles, then $\left\{z_{1}, z_{2}\right\},\left\{z_{1}, a\right\},\left\{z_{2}, b\right\} \notin E(H)$. Hence, $C$ is an induced cycle in $H$. On the other hand, if there exists $v \in V(H)$ such that $d(v, C) \geqslant 2$, then $H^{\prime}=H \backslash N_{G}[v]$ is a c-minor of $G$ and $C \subseteq H^{\prime} \subset H$. This is a contradiction by the minimality of $H$. Therefore, $d(v, C) \leqslant 1$ for each $v \in V(H)$.

Now, if $\operatorname{deg}_{H}(b) \geqslant 3$, then there exists $c \in V(H) \backslash V(C)$ such that $\{b, c\} \in E(H)$. If $\left\{c, z_{2}\right\} \notin E(G)$ implies that $N_{H_{1}}\left(z_{2}\right)$ has two free vertices, $w_{2}$ and $x$, in $H_{1}=H \backslash N_{H}\left[w_{1}, c\right]$, this is a contradiction by Lemma 22. Thus $\left\{c, z_{2}\right\} \in E(H)$. Furthermore, $\{a, c\},\left\{z_{1}, c\right\} \notin$ $E(H)$ since $(a, b, c)$ and $\left(z_{1}, w_{1}, a, b, c\right)$ are not cycles in $G$. Hence, if $\operatorname{deg}_{H}(c) \geqslant 3$, then there exists $d \in V(H) \backslash V(C)$ such that $\{c, d\} \in E(H)$. Also, $\{d, b\},\left\{d, z_{2}\right\},\left\{d, z_{1}\right\} \notin E(H)$ since $(c, b, d),\left(z_{2}, d, c\right)$ and $\left(z_{1}, x, z_{2}, c, d\right)$ are not cycles of $G$. But $d(d, C) \leqslant 1$, so $\{a, d\} \in$ $E(H)$. Consequently, $N_{H_{2}}\left(z_{1}\right)$ has two free vertices, $w_{1}$ and $x$, in $H_{2}=H \backslash N_{H}\left[d, w_{2}\right]$, a contradiction by Lemma 22 , then $\operatorname{deg}_{H}(c)=2$. This implies, $N_{H_{3}}\left(z_{2}\right)$ has two free vertices, $w_{2}$ and $c$, in $H_{3}=H \backslash N_{H}[a]$. This is not possible, therefore $\operatorname{deg}_{H}(b)=2$. Similarly, $\operatorname{deg}_{H}(a)=2$.

Now, if $\operatorname{deg}_{H}\left(z_{2}\right) \geqslant 3$ we have that there exists $c^{\prime} \in V(H) \backslash V(C)$ such that $\left\{c^{\prime}, z_{2}\right\} \in$ $E(H)$. If there exists $d^{\prime} \in V(H) \backslash V(C)$ such that $\left\{c^{\prime}, d^{\prime}\right\} \in E(H)$, then $\left\{d^{\prime}, z_{1}\right\}$ or $\left\{d^{\prime}, z_{2}\right\} \in E(G)$, since $d\left(d^{\prime}, C\right) \leqslant 1$. But $\left(c^{\prime}, d^{\prime}, z_{2}\right)$ and $\left(x, z_{2}, c^{\prime}, d^{\prime}, z_{1}\right)$ are not cycles 
of $H$, thus, $N_{H}\left(c^{\prime}\right) \subseteq\left\{z_{1}, z_{2}\right\}$. Consequently, $N_{H_{4}}\left(z_{2}\right)$ has two free vertices, $x$ and $c^{\prime}$, in $H_{4}=H \backslash N_{H}\left[w_{1}\right]$, a contradiction. Hence $\operatorname{deg}_{H}\left(z_{2}\right)=2$. Similarly, $\operatorname{deg}_{H}\left(z_{1}\right)=2$. Furthermore, since $H$ is minimal, then it is connected. Therefore, $H=C$ and $C$ is a c-minor of $G$.

\section{König and Cohen-Macaulay graphs without 3-cycles and 5- cycles}

Definition 25. A simplicial complex $\Delta$ is shellable if the facets (maximal faces) of $\Delta$ can be ordered $F_{1}, \ldots, F_{s}$ such that for all $1 \leqslant i<j \leqslant s$, there exists some $v \in F_{j} \backslash F_{i}$ and some $l \in\{1, \ldots, j-1\}$ with $F_{j} \backslash F_{l}=\{v\}$. In this case, $F_{1}, \ldots, F_{s}$ is called a shelling of $\Delta$. A graph $G$ is called shellable if $\Delta_{G}$ is shellable. Furthermore, the facet set of $\Delta$ is denoted by $\mathcal{F}(\Delta)$.

Remark 26. The following properties: shellable, Cohen-Macaulay, sequentially CohenMacaulay and vertex decomposable are closed under c-minors (see [1], [20]).

Remark 27 . If $G$ is very well-covered with a perfect matching $e_{1}, \ldots, e_{g}$, then the following conditions are equivalent:

(1) $G$ is Cohen-Macaulay.

(2) There are no 4-cycles with two $e_{i}$ 's.

Proof. By ([5], Theorem 3.4).

Proposition 28. Let $G$ be a König graph where $G^{\prime}=G \backslash Z_{G}$. Then the following properties are equivalent:

(i) $G$ is unmixed vertex decomposable.

(ii) $\Delta_{G}$ is pure shellable.

(iii) $R / I(G)$ is Cohen-Macaulay.

(iv) $V\left(G^{\prime}\right)=\varnothing$ or $G^{\prime}$ is an unmixed graph with a perfect matching $e_{1}, \ldots, e_{g}$ of König type without 4-cycles with two $e_{i}$ 's.

(v) $V\left(G^{\prime}\right)=\varnothing$ or there exists a relabelling of the vertices $V\left(G^{\prime}\right)=\left\{x_{1}, \ldots, x_{h}, y_{1}, \ldots, y_{h}\right\}$ such that $\left\{x_{1}, y_{1}\right\}, \ldots,\left\{x_{h}, y_{h}\right\}$ is a perfect matching, $X=\left\{x_{1}, \ldots, x_{h}\right\}$ is a minimal vertex cover of $G^{\prime}$ and the following conditions holds:

(a) If $a_{i} \in\left\{x_{i}, y_{i}\right\}$ and $\left\{a_{i}, x_{j}\right\},\left\{y_{j}, x_{k}\right\} \in E\left(G^{\prime}\right)$, then $\left\{a_{i}, x_{k}\right\} \in E\left(G^{\prime}\right)$ for $i \neq j$ and $j \neq k$;

(b) If $\left\{x_{i}, y_{j}\right\} \in E\left(G^{\prime}\right)$, then $i \leqslant j$. 
Proof. (i) $\Leftrightarrow$ (ii) $\Leftrightarrow$ (iii) In each case $G$ is unmixed and König. Hence, by Lemma 17, $G$ is totally disconnected or $G^{\prime}$ is very well-covered. If $G$ is totally disconnected, then we obtain the equivalences. Now, if $G^{\prime}$ is very well-covered, then by ([13], Theorem 1.1) we obtain the equivalences.

(iv) $\Rightarrow$ (iii) We can assume that $V\left(G^{\prime}\right) \neq \varnothing$. Thus, by Lemma $17, G^{\prime}$ is very wellcovered. Hence, by Remark $27 G^{\prime}$ is Cohen-Macaulay. Therefore, $G$ is Cohen-Macaulay.

(iii) $\Rightarrow(v)$ Since $R / I(G)$ is Cohen-Macaulay, then $G$ is unmixed. Consequently, by Lemma 17, we can assume that $G^{\prime}$ is very well-covered. Hence, by ([13], Lemma 3.1), $G^{\prime}$ satisfies (v).

$(\mathrm{v}) \Rightarrow(\mathrm{iv})$ We can assume that $V\left(G^{\prime}\right) \neq \varnothing$. Since, $e_{1}=\left\{x_{1}, y_{1}\right\}, \ldots, e_{h}=\left\{x_{h}, y_{h}\right\}$ is a perfect matching, then $\nu\left(G^{\prime}\right)=h$. Furthermore, $X$ is a minimal vertex cover, so $\tau\left(G^{\prime}\right)=h$. Hence, $e_{1}, \ldots, e_{h}$ is a perfect matching of König type. Thus, from (a) and Proposition $15, G^{\prime}$ is unmixed. On the other hand $\left\{y_{1}, \ldots, y_{h}\right\}$ is a stable set. Therefore, from (b), there are no 4-cycles with two $e_{i}$ 's.

Corollary 29. Let $G$ be a connected König graph. If $G$ is Cohen-Macaulay, then $G$ is an isolated vertex or $G$ has at least one free vertex.

Proof. By Proposition 28, if $G$ is not an isolated vertex, then $G$ has a perfect matching $e_{1}=\left\{x_{1}, y_{1}\right\}, \ldots, e_{h}=\left\{x_{h}, y_{h}\right\}$ where $\left\{x_{1}, \ldots, x_{h}\right\}$ is a minimal vertex cover. Thus, $\left\{y_{1}, \ldots, y_{h}\right\}$ is a maximal stable set. Furthermore, if $\left\{x_{i}, y_{j}\right\}$, then $i \leqslant j$. Hence, $N_{G}\left(y_{1}\right)=$ $\left\{x_{1}\right\}$. Therefore, $y_{1}$ is a free vertex.

Lemma 30. Let $G$ be an unmixed connected graph with a perfect matching $e_{1}, \ldots, e_{g}$ of König type without 4 -cycles with two $e_{i}$ 's and $g \geqslant 2$. For each $z \in V(G)$ we have that:

(a) If $\operatorname{deg}_{G}(z) \geqslant 2$, then there exist $\left\{z, w_{1}\right\},\left\{w_{1}, w_{2}\right\} \in E(G)$ such that $\operatorname{deg}_{G}\left(w_{2}\right)=1$. Furthermore, $e_{i}=\left\{w_{1}, w_{2}\right\}$ for some $i \in\{1, \ldots, g\}$.

(b) If $\operatorname{deg}_{G}(z)=1$, then there exist $\left\{z, w_{1}\right\},\left\{w_{1}, w_{2}\right\},\left\{w_{2}, w_{3}\right\} \in E(G)$ such that $\operatorname{deg}_{G}\left(w_{3}\right)$ $=1$. Moreover, $e_{i}=\left\{z, w_{1}\right\}$ and $e_{j}=\left\{w_{2}, w_{3}\right\}$ for some $i, j \in\{1, \ldots, g\}$.

Proof. Since $e_{1}=\left\{x_{1}, y_{1}\right\}, \ldots, e_{g}=\left\{x_{g}, y_{g}\right\}$ is a perfect matching of König type we can assume $D=\left\{x_{1}, \ldots, x_{g}\right\}$ is a minimal vertex cover. Thus, $F=\left\{y_{1}, \ldots, y_{g}\right\}$ is a maximal stable set. By Proposition 28, we can assume that if $\left\{x_{i}, y_{j}\right\} \in E(G)$, then $i \leqslant j$. Now, we take a vertex $z \in V(G)$.

(a) First, we suppose that $z=x_{k}$ and there is a vertex $x_{j}$ in $N_{G}\left(x_{k}\right)$. If $y_{j}$ is a free vertex, then we take $w_{1}=x_{j}$ and $w_{2}=y_{j}$, and $e_{j}=\left\{w_{1}, w_{2}\right\}$. Now, we can assume $N_{G}\left(y_{j}\right) \backslash x_{j}=\left\{x_{p_{1}}, \ldots, x_{p_{r}}\right\}$ with $p_{1}<\cdots<p_{r}<j$. If $y_{p_{1}}$ is not a free vertex, then there is a vertex $x_{p}$ with $p<p_{1}$ such that $\left\{x_{p}, y_{p_{1}}\right\} \in E(G)$. Since $G$ is unmixed, from Proposition 15, we obtain that $\left\{x_{p}, y_{j}\right\}=\left(\left\{x_{p}, y_{p_{1}}\right\} \backslash y_{p_{1}}\right) \cup\left(\left\{y_{j}, x_{p_{1}}\right\} \backslash x_{p_{1}}\right) \in E(G)$. But $p<p_{1}$, a contradiction since $p_{1}$ is minimal. Consequently, $\operatorname{deg}_{G}\left(y_{p_{1}}\right)=1$. Also, from Proposition 15, we have that $\left\{x_{k}, x_{p_{1}}\right\}=\left(\left\{x_{k}, x_{j}\right\} \backslash x_{j}\right) \cup\left(\left\{x_{p_{1}}, y_{j}\right\} \backslash y_{j}\right) \in E(G)$. Hence, we take $w_{1}=x_{p_{1}}$ and $w_{2}=y_{p_{1}}$, and we have that $e_{p_{1}}=\left\{w_{1}, w_{2}\right\}$. Now, we 
assume that $z=x_{k}$ and $N_{G}\left(x_{k}\right) \backslash y_{k}=\left\{y_{j_{1}}, \ldots, y_{j_{t}}\right\}$ with $k<j_{1}<\cdots<j_{t}$. We suppose that $\operatorname{deg}_{G}\left(x_{j_{t}}\right) \geqslant 2$. If there is a vertex $y_{r}$ such that $\left\{x_{j_{t}}, y_{r}\right\} \in E(G)$, then $r>j_{t}$. Since $G$ is unmixed, $\left\{x_{k}, y_{r}\right\}=\left(\left\{x_{k}, y_{j_{t}}\right\} \backslash y_{j_{t}}\right) \cup\left(\left\{y_{r}, x_{j_{t}}\right\} \backslash x_{j_{t}}\right) \in E(G)$, a contradiction since $j_{t}$ is maximal. Thus, there exists a vertex $x_{p}$ such that $\left\{x_{j_{t}}, x_{p}\right\} \in E(G)$. But, since $G$ is unmixed, then $\left\{x_{k}, x_{p}\right\}=\left(\left\{x_{k}, y_{j_{t}}\right\} \backslash y_{j_{t}}\right) \cup\left(\left\{x_{p}, x_{j_{t}}\right\} \backslash x_{j_{t}}\right) \in E(G)$. This is a contradiction since $N_{G}\left(x_{k}\right) \backslash y_{k}=\left\{y_{j_{1}}, \ldots, y_{i_{t}}\right\}$. Consequently, $\operatorname{deg}_{G}\left(x_{j_{t}}\right)=1$. Therefore, we take $w_{1}=y_{j_{t}}$ and $w_{2}=x_{j_{t}}$, with $e_{j_{t}}=\left\{w_{1}, w_{2}\right\}$.

Finally, we assume that $z=y_{k}$, since $y_{k}$ is not a free vertex, then $N_{G}\left(y_{k}\right) \backslash x_{k}=$ $\left\{x_{j_{1}}, \ldots, x_{j_{r}}\right\}$ with $j_{1}<\cdots<j_{r}<k$. If $y_{j_{1}}$ is not a free vertex, then there is a vertex $x_{q}$ such that $\left\{x_{q}, y_{j_{1}}\right\} \in E(G)$ with $q<j_{1}$. This implies $\left\{x_{q}, y_{k}\right\}=\left(\left\{x_{q}, y_{j_{1}}\right\} \backslash y_{j_{1}}\right) \cup$ $\left(\left\{x_{j_{1}}, y_{k}\right\} \backslash x_{j_{1}}\right) \in E(G)$. But $q<j_{1}$, a contradiction. Therefore, $\operatorname{deg}_{G}\left(y_{j_{1}}\right)=1$ and we take $w_{1}=x_{j_{1}}$ and $w_{2}=y_{j_{1}}$. Hence, $e_{j_{1}}=\left\{w_{1}, w_{2}\right\}$.

(b) Since $e_{1}, \ldots, e_{g}$ is a perfect matching, then there exists $i \in\{1, \ldots, g\}$ such that $e_{i}=\left\{z, z^{\prime}\right\}$. Since $G$ is connected, $z$ is a free vertex and $g \geqslant 2$, then $\operatorname{deg}_{G}\left(z^{\prime}\right) \geqslant 2$. Thus, by (a) there exist $w_{1}^{\prime}, w_{2}^{\prime} \in V(G)$ such that $\left\{z^{\prime}, w_{1}^{\prime}\right\},\left\{w_{1}^{\prime}, w_{2}^{\prime}\right\} \in E(G)$ where $\operatorname{deg}_{G}\left(w_{2}^{\prime}\right)=1$ and $\left\{w_{1}^{\prime}, w_{2}^{\prime}\right\}=e_{j}$ for some $j \in\{1, \ldots, g\}$. Therefore, we take $w_{1}=z^{\prime}, w_{2}=w_{1}^{\prime}, w_{3}=w_{2}^{\prime}$. Consequently, $e_{i}=\left\{z, w_{1}\right\}$ and $e_{j}=\left\{w_{2}, w_{3}\right\}$.

Remark 31. If $C_{n}$ is a n-cycle, then $C_{n}$ is vertex decomposable, shellable or sequentially Cohen-Macaulay if and only if $n=3$ or 5 (see [9] and [22]). Furthermore, a chordal graph, which is a graph whose induced cycles are 3 -cycles, is vertex decomposable (see Corollary 7 in [22]). In particular trees are vertex decomposable.

Theorem 32. Let $G$ be a graph without 3-cycles and 5-cycles. If $G_{1}, \ldots, G_{k}$ are the connected components of $G$, then the following conditions are equivalent:

(a) $G$ is unmixed vertex decomposable.

(b) $G$ is pure shellable.

(c) $G$ is Cohen-Macaulay

(d) $G$ is unmixed and if $G_{i}$ is not an isolated vertex, then $G_{i}$ has a perfect matching $e_{1}, \ldots, e_{g}$ of König type without 4 -cycles with two $e_{i}^{\prime} s$.

Proof. (a) $\Rightarrow$ (b) $\Rightarrow$ (c) (see [16], [20], [22]).

(d) $\Rightarrow$ (a) Since each component $G_{i}$ is König, then $G$ is König. Therefore, from Proposition 28, $G$ is unmixed vertex decomposable.

$(\mathrm{c}) \Rightarrow(\mathrm{d})$ Since $G$ is Cohen-Macaulay, then $G$ is unmixed. We proceed by induction on $|V(G)|$. We take $x \in V(G)$ such that $\operatorname{deg}_{G}(x)$ is minimal and we suppose that $N_{G}(x)=$ $\left\{z_{1}, \ldots, z_{r}\right\}$. By Remark 26, $G^{\prime}=G \backslash N_{G}[x]$ is a Cohen-Macaulay graph. We take $G_{1}^{\prime}, \ldots, G_{s}^{\prime}$, the connected components of $G^{\prime}$. We can assume that $V\left(G_{i}^{\prime}\right)=\left\{y_{i}\right\}$ for $i \in\left\{1, \ldots, s^{\prime}\right\}$. Since $\operatorname{deg}_{G}(x)$ is minimal, this implies $\left\{y_{i}, z_{j}\right\} \in E(G)$ for all $i \in\left\{1, \ldots, s^{\prime}\right\}$ and $j \in\{1, \ldots, r\}$. Since $G$ does not contain 3 -cycles, we have that $N_{G}(x)$ is a stable 
set. If $s^{\prime}=s$, then the only maximal stable sets of $G$ are $\left\{y_{1}, \ldots, y_{s^{\prime}}, x\right\}$ and $\left\{z_{1}, \ldots, z_{r}\right\}$. Thus, $G$ is a bipartite graph. So, $G$ is König. Hence, by Proposition 28, $G$ satisfies (d). Consequently, we can assume $s>s^{\prime}$, implying that there is a component $G_{i}^{\prime}$ with an edge $e=\left\{w, w^{\prime}\right\}$.

Now, we suppose that $r \geqslant 2$. Since $\operatorname{deg}_{G}(x)$ is minimal there exist $a, b \in V(G)$ such that $\{a, w\},\left\{b, w^{\prime}\right\} \in E(G)$. If $a=b$, then $\left(a, w, w^{\prime}\right)$ is a 3-cycle in $G$. Hence, $a \neq b$. If $a, b \in N_{G}(x)$, then $\left(x, a, w, w^{\prime}, b\right)$ is a 5-cycle in $G$. Thus, $\left|\left\{w, w^{\prime}, a, b\right\} \cap V\left(G_{i}^{\prime}\right)\right| \geqslant 3$. By induction hypothesis, $G^{\prime}$ satisfies (d). So, $G_{i}^{\prime}$ has a perfect matching and $\tau\left(G_{i}^{\prime}\right) \geqslant 2$. Furthermore, by Corollary 29, $G_{i}^{\prime}$ has a free vertex $a^{\prime}$. Then, by Lemma 30 (b), there exist edges $\left\{a^{\prime}, w_{1}\right\},\left\{w_{1}, w_{2}\right\},\left\{w_{2}, b^{\prime}\right\} \in E\left(G_{i}^{\prime}\right)$ such that $\operatorname{deg}_{G_{i}^{\prime}}\left(a^{\prime}\right)=\operatorname{deg}_{G_{i}^{\prime}}\left(b^{\prime}\right)=1$. By the minimality of $\operatorname{deg}_{G}(x)$ we have that $a^{\prime}$ and $b^{\prime}$ are adjacent with at least $r-1$ neighbor vertices of $x$. If $r \geqslant 3$, then there exists $z_{j}$ such that $z_{j} \in N_{G}\left(a^{\prime}\right) \cap N_{G}\left(b^{\prime}\right)$. This implies that $\left(a^{\prime}, w_{1}, w_{2}, b^{\prime}, z_{j}\right)$ is a 5 -cycle of $G$. But $G$ does not have 5 -cycles, consequently, $r=2$. We can assume that $\left\{a^{\prime}, z_{1}\right\},\left\{b^{\prime}, z_{2}\right\} \in E(G)$, implying $C=\left(x, z_{1}, a^{\prime}, w_{1}, w_{2}, b^{\prime}, z_{2}\right)$ is a 7-cycle with $\operatorname{deg}_{G}\left(a^{\prime}\right)=\operatorname{deg}_{G}\left(b^{\prime}\right)=\operatorname{deg}_{G}(x)=2$. Hence, by Proposition 24, $C$ is a c-minor of $G$. Thus, by Remark 26, $C$ is Cohen-Macaulay. This is a contradiction by Remark 31 . Therefore, $\operatorname{deg}_{G}(x)=r \leqslant 1$.

If $r=0$, then the result is clear. Now, if $r=1$ we can assume that $G_{1}, \ldots, G_{k}$ are the connected components of $G$ and $z_{1} \in V\left(G_{1}\right)$. Consequently, the connected components of $G \backslash N_{G}[x]$ are $F_{1}, \ldots, F_{l}, G_{2}, \ldots, G_{k}$ where $F_{1}, \ldots, F_{l}$ are the connected components of $G_{1} \backslash N_{G_{1}}[x]$. By induction hypothesis $G_{2}, \ldots, G_{k}$ satisfy (d). If $F_{j}=\left\{d_{j}\right\}$, then $N_{G}\left(z_{1}\right)$ has two free vertices, $d_{j}$ and $x$, a contradiction by Lemma 22 . Hence, $\left|V\left(F_{i}\right)\right| \geqslant 2$ for $i \in\{1, \ldots, l\}$. By induction hypothesis, we have that $F_{i}$ has a perfect matching $M_{i}=\left\{e_{1}^{i}, \ldots, e_{g_{i}}^{i}\right\}$ of König type. Thus, $\{e\} \cup\left(\bigcup_{i=1}^{l} M_{i}\right)$ is a perfect matching of $G_{1}$, where $e=\left\{x, z_{1}\right\}$. Also, $\left\{z_{1}\right\} \cup\left(\bigcup_{i=1}^{l} X_{i}\right)$ is a vertex cover of $G_{1}$, where $X_{i}$ is a minimal vertex cover of $F_{i}$. Consequently, $\nu\left(G_{1}\right) \geqslant 1+\sum_{i=1}^{l}\left|M_{i}\right|=1+\sum_{i=1}^{l} g_{i}=1+\sum_{i=1}^{l}\left|X_{i}\right| \geqslant \tau\left(G_{1}\right)$. This implies that $G_{1}$ is König. Furthermore, by Remark 26, we have that $G_{1}$ is CohenMacaulay. Therefore, by Proposition 28, $G_{1}$ satisfies (d).

Corollary 33. Let $G$ be a connected graph without 3-cycles and 5-cycles. If $G$ is CohenMacaulay, then $G$ has at least one extendable vertex $x$ adjacent to a free vertex.

Proof. From Theorem 32, $G$ is König. Thus, by Corollary 29 there exists a free vertex $x$. If $N_{G}(x)=\{y\}$, then by Remark $7, y$ is a shedding vertex. Therefore, from Corollary 13, $y$ is an extendable vertex, since $G$ is unmixed.

Definition 34. $G$ is called whisker graph if there exists an induced subgraph $H$ of $G$ such that $V(H)=\left\{x_{1}, \ldots, x_{s}\right\}, V(G)=V(H) \cup\left\{y_{1}, \ldots, y_{s}\right\}$ and $E(G)=E(H) \cup W(H)$ where $W(H)=\left\{\left\{x_{1}, y_{1}\right\}, \ldots,\left\{x_{s}, y_{s}\right\}\right\}$. The edges of $W(H)$ are called whiskers and they form a perfect matching.

Definition 35. The girth of $G$ is the length of the smallest cycle or infinite if $G$ is a forest. 
Corollary 36. Let $G$ be a connected graph of girth 6 or more. If $G$ is not an isolated vertex, then the following conditions are equivalent:

(i) $G$ is unmixed vertex decomposable.

(ii) $\Delta_{G}$ is pure shellable.

(iii) $R / I(G)$ is Cohen-Macaulay.

(iv) $G$ is an unmixed König graph.

(v) $G$ is very well-covered.

(vi) $G$ is unmixed with $G \neq C_{7}$.

(vii) $G$ is a whisker graph.

Proof. (i) $\Rightarrow$ (ii) $\Rightarrow$ (iii) (see [16], [20], [22]). (iii) $\Rightarrow$ (iv) $G$ is unmixed and from Theorem $32, G$ is König. (iv) $\Rightarrow$ (v) From Lemma 17 . (v) $\Rightarrow$ (vi) It is clear, since $C_{7}$ is not very well-covered.

$($ vi $) \Rightarrow$ (vii) By ([8], Corollary 5), the pendant edges $\left\{x_{1}, y_{1}\right\}, \ldots,\left\{x_{g}, y_{g}\right\}$ of $G$ form a perfect matching. Since $\left\{x_{i}, y_{i}\right\}$ is a pendant edge, we can assume that $\operatorname{deg}_{G}\left(y_{i}\right)=1$ for each $1 \leqslant i \leqslant g$. We take $H=G\left[x_{1}, \ldots, x_{n}\right]$. Therefore, $G$ is a whisker graph with $W(H)=\left\{\left\{x_{1}, y_{1}\right\}, \ldots,\left\{x_{g}, y_{g}\right\}\right\}$

(vii) $\Rightarrow$ (i) By ([6], Theorem 4.4).

\section{$5 \quad$ Vertex decomposable and shellable properties in graphs with- out 3-cycles and 5-cycles}

Definition 37. A 5-cycle $C$ of $G$ is called basic if $C$ does not contain two adjacent vertices of degree three or more in $G$.

Lemma 38. If $G$ is a graph, then any vertex of degree at least 3 in a basic 5-cycle is a shedding vertex.

Proof. Let $C=\left(x_{1}, x_{2}, x_{3}, x_{4}, x_{5}\right)$ be a basic 5-cycle. We suppose that $\operatorname{deg}_{G}\left(x_{1}\right) \geqslant 3$, since $C$ is a basic 5-cycle, then $\operatorname{deg}_{G}\left(x_{2}\right)=\operatorname{deg}_{G}\left(x_{5}\right)=2$. Also, we can assume that $\operatorname{deg}_{G}\left(x_{3}\right)=2$. We take a stable set $S$ of $G \backslash N_{G}\left[x_{1}\right]$. Since $\left\{x_{3}, x_{4}\right\} \in E(G)$, then $\left|S \cap\left\{x_{3}, x_{4}\right\}\right| \leqslant 1$. Hence, $x_{3} \notin S$ or $x_{4} \notin S$. Consequently, $S \cup\left\{x_{2}\right\}$ or $S \cup\left\{x_{5}\right\}$ is a stable set of $G \backslash x_{1}$. Therefore, $x_{1}$ is a shedding vertex.

Remark 39. If $G$ has a shedding vertex $v$ where $G \backslash v$ and $G \backslash N_{G}[v]$ are shellable with shelling $F_{1}, \ldots, F_{k}$ and $G_{1}, \ldots, G_{q}$, respectively, then $G$ is shellable with shelling $F_{1}, \ldots, F_{k}, G_{1} \cup\{v\}, \ldots, G_{q} \cup\{v\}$ (see Lemma 6 in [21]). 
Theorem 40. Let $G$ be a connected graph with a basic 5-cycle $C . G$ is a shellable graph if and only if there is a shedding vertex $x \in V(C)$ such that $G \backslash x$ and $G \backslash N_{G}[x]$ are shellable graphs.

Proof. $\Rightarrow)$ We can suppose that $C=\left(x_{1}, x_{2}, x_{3}, x_{4}, x_{5}\right)$. If $G=C$, then $G$ is shellable. By Remark 9, each vertex is a shedding vertex. Furthermore, $G \backslash x_{1}$ is a path with shelling $\left\{x_{2}, x_{4}\right\},\left\{x_{2}, x_{5}\right\},\left\{x_{3}, x_{5}\right\}$ and $G \backslash N_{G}\left[x_{1}\right]$ is an edge. Therefore, $G \backslash x_{1}$ and $G \backslash N_{G}\left[x_{1}\right]$ are shellable graphs. Now, we suppose $G \neq C$. We can assume that $\operatorname{deg}_{G}\left(x_{1}\right) \geqslant 3$. Since $C$ is a basic 5-cycle, then $\operatorname{deg}_{G}\left(x_{2}\right)=\operatorname{deg}_{G}\left(x_{5}\right)=2$. Also, we can suppose $\operatorname{deg}_{G}\left(x_{3}\right)=2$ and $\operatorname{deg}_{G}\left(x_{4}\right) \geqslant 2$. By Lemma $38, x_{1}$ is a shedding vertex. Furthermore by Remark 26 , we have that $G \backslash N_{G}\left[x_{1}\right]$ is a shellable graph. Now, we will prove that $G_{1}=G \backslash x_{1}$ is shellable. Since $G$ is shellable and since shellability is closed under c-minors, then $G_{2}=G \backslash N_{G}\left[x_{2}\right]$ is shellable. We assume that $F_{1}, \ldots, F_{r}$ is a shelling of $\Delta_{G_{2}}$. Also, $G_{3}=G \backslash N_{G}\left[x_{3}, x_{5}\right]$ is shellable. We suppose that $H_{1}, H_{2}, \ldots, H_{k}$ is shelling of $\Delta_{G_{3}}$. We take $F \in \mathcal{F}\left(\Delta_{G_{1}}\right)$. If $x_{2} \in F$, then $F \backslash x_{2} \in \mathcal{F}\left(\Delta_{G_{2}}\right)$ and there exists $F_{i}$ such that $F=F_{i} \cup\left\{x_{2}\right\}$. If $x_{2} \notin F$, then $x_{3} \in F$ and $x_{4} \notin F$. Thus, $x_{5} \in F$. Hence, $F \backslash\left\{x_{3}, x_{5}\right\} \in \mathcal{F}\left(\Delta_{G_{3}}\right)$, then there exists $H_{j}$ such that $F=H_{j} \cup\left\{x_{3}, x_{5}\right\}$. This implies, $\mathcal{F}\left(\Delta_{G_{1}}\right)=\left\{F_{1} \cup\left\{x_{2}\right\}, \ldots, F_{r} \cup\left\{x_{2}\right\}, H_{1} \cup\left\{x_{3}, x_{5}\right\}, \ldots, H_{k} \cup\left\{x_{3}, x_{5}\right\}\right\}$. Furthermore, $F_{1} \cup\left\{x_{2}\right\}, \ldots, F_{r} \cup\left\{x_{2}\right\}$ and $H_{1} \cup\left\{x_{3}, x_{5}\right\}, \ldots, H_{k} \cup\left\{x_{3}, x_{5}\right\}$ are shellings. Now, $x_{3} \in\left(H_{j} \cup\right.$ $\left.\left\{x_{3}, x_{5}\right\}\right) \backslash\left(F_{i} \cup\left\{x_{2}\right\}\right)$ and $H_{j}$ is a stable set of $G$ without vertices of $C$. So, $H_{j} \cup\left\{x_{2}, x_{5}\right\}$ is a maximal stable set of $G_{1}$ since $N_{G}\left(x_{2}, x_{5}\right)=V(C)$ and $\left\{x_{2}, x_{5}\right\} \notin E(G)$. Consequently, $H_{j} \cup\left\{x_{2}, x_{5}\right\}=F_{l} \cup\left\{x_{2}\right\}$ for some $l \in\{1, \ldots, r\}$ and $\left(H_{j} \cup\left\{x_{3}, x_{5}\right\}\right) \backslash\left(F_{l} \cup\left\{x_{2}\right\}\right)=\left\{x_{3}\right\}$. Therefore, $G_{1}$ is a shellable graph.

$\Leftarrow)$ By Remark 39 .

Definition 41. A cut vertex of a graph is one whose removal increases the number of connected components. A block of a graph is a maximal subgraph without cut vertices. A connected graph without cut vertices with at least three vertices is called 2-connected graph.

In the following result $P$ is a property closed under c-minors.

Theorem 42. Let $G$ be a graph without 3-cycles and 5-cycles with a 2-connected block $B$. If $G$ satisfies the property $P$ and $B$ does not satisfy $P$, then there exists $x \in D_{1}(B)$ such that $\operatorname{deg}_{G}(x)=1$.

Proof. By contradiction, we assume that if $x \in D_{1}(B)$, then $\left|N_{G}(x)\right|>1$. Thus, there exist $a, b \in N_{G}(x)$ with $a \neq b$. We can suppose that $a \in V(B)$. If $b \in V(B)$, then $G[\{x\} \cup V(B)]$ is 2 -connected. But $B \subsetneq G[\{x\} \cup V(B)]$. This is a contradiction since $B$ is a block. Consequently, $V(B) \cap N_{G}(x)=\{a\}$. Now, we suppose that $b \in D_{1}(B)$. Since there is no 3-cycle in $G$, then $a \notin N_{G}(b)$. Hence, there exists $c \in N_{G}(b) \cap V(B)$ such that $c \neq a$. This implies $G[\{x, b\} \cup V(B)]$ is 2-connected. But $B \subsetneq G[\{x, b\} \cup V(B)]$, a contradiction. Then $D_{1}(B) \cap N_{G}(x)=\varnothing$. Thus, $N_{G}(x) \cap\left(V(B) \cup D_{1}(B)\right)=\{a\}$ and $b \in D_{2}(B)$. Now, if $D_{1}(B)=\left\{x_{1}, \ldots, x_{r}\right\}$, then there exists an $a_{i}$ such that $V(B) \cap N_{G}\left(x_{i}\right)=\left\{a_{i}\right\}$. Also, there exists $b_{i}$ such that $b_{i} \in N_{G}\left(x_{i}\right) \cap D_{2}(B)$. We can suppose that $L=\left\{b_{1}, \ldots, b_{s}\right\}=$ 
$\left\{b_{1}, \ldots, b_{r}\right\}$ with $b_{i} \neq b_{j}$ for $1 \leqslant i<j \leqslant s$. We will prove that $L$ is a stable set. Suppose that $\left\{b_{i}, b_{j}\right\} \in E(G)$, if $a_{i}=a_{j}$, then $\left(a_{i}, x_{i}, b_{i}, b_{j}, x_{j}, a_{i}\right)$ is a 5 -cycle in $G$, this is a contradiction. Consequently $a_{i} \neq a_{j}$ and the induced subgraph $G\left[\left\{x_{i}, b_{i}, b_{j}, x_{j}\right\} \cup V(B)\right]$ is 2-connected. But $B$ is a block, then $\left\{b_{i}, b_{j}\right\} \notin E(G)$. Therefore, $L$ is a stable set. Furthermore, $G^{\prime}=G \backslash N_{G}[L]$ is a c-minor of $G$, implying that $G^{\prime}$ satisfies the property $P$. Since $D_{1}(B) \subset N_{G}(L)$, we have that $B$ is a connected component of $G^{\prime}$. But, $B$ does not satisfy $P$. This is a contradiction since each connected component of $G$ is a c-minor. Therefore, there exists a free vertex in $D_{1}(B)$.

Corollary 43. Let $G$ be a graph without 3-cycles and 5-cycles and $B$ a 2-connected block. If $G$ is shellable (unmixed, Cohen-Macaulay, sequentially Cohen-Macaulay or vertex decomposable) and $B$ is not shellable (unmixed, Cohen - Macaulay, sequentially CohenMacaulay or vertex decomposable), then there exists $x \in D_{1}(B)$ such that $\operatorname{deg}_{G}(x)=1$.

Proof. From Remark 20, Remark 26 and Theorem 42.

Corollary 44. Let $G$ be a bipartite graph and $B$ a 2-connected block. If $G$ is shellable, then there exists $x \in D_{1}(B)$ such that $\operatorname{deg}_{G}(x)=1$.

Proof. Since $G$ is bipartite, then $B$ is bipartite. If $H$ is a shellable bipartite graph, then $H$ has a free vertex (see [18], Lemma 2.8), and so $H$ is not 2-connected. Hence, $B$ is not shellable. Therefore, by Corollary 43 , there exists $x \in D_{1}(B)$ such that $\operatorname{deg}_{G}(x)=1$.

Lemma 45. Let $G$ be a graph without 3-cycles and 5-cycles. If $G$ is vertex decomposable, then $G$ has a free vertex.

Proof. Since $G$ is vertex decomposable, then there is a shedding vertex $x$. Furthermore, there are no 5-cycles in $G$. Hence, by Theorem 5, there exists $y \in N_{G}(x)$ such that $N_{G}[y] \subseteq N_{G}[x]$. If $z \in N_{G}(y) \backslash x$, then $(x, y, z)$ is a 3 -cycle. This is a contradiction. Therefore, $N_{G}(y)=\{x\}$, implying that $y$ is a free vertex.

Theorem 46. Let $G$ be a graph without 3-cycles and 5-cycles. $G$ is vertex decomposable if and only if there exists a free vertex $x$ with $N_{G}(x)=\{y\}$ such that $G_{1}=G \backslash N_{G}[x]$ and $G_{2}=G \backslash N_{G}[y]$ are vertex decomposable.

Proof. $\Rightarrow)$ By Lemma 45 there exists a free vertex $x$. Furthermore, by Remark 26, $G_{1}$ and $G_{2}$ are vertex decomposable.

$\Leftarrow)$ By Remark 7, $y$ is a shedding vertex. Moreover, $G \backslash y=G_{1} \cup\{x\}$. Furthermore, since $G_{1}$ is vertex decomposable, then $G \backslash y$ is also it. Therefore, $G$ is vertex decomposable, since $G_{2}$ is vertex decomposable.

Corollary 47. If $G$ is a 2-connected graph without 3-cycles and 5-cycles, then $G$ is not a vertex decomposable.

Proof. Since $G$ is 2-connected, then $G$ does not have a free vertex. Therefore, by Lemma $45, G$ is not vertex decomposable. 
Theorem 48. Let $G$ be a vertex decomposable graph without 3-cycles and 5-cycles. If $B$ is a 2-connected block of $G$, then $D_{1}(B)$ has a free vertex.

Proof. By Corollary 47, $B$ is not vertex decomposable. Therefore, by Theorem $42, D_{1}(B)$ has a free vertex.

Definition 49. Let $G_{1}, G_{2}$ be graphs. If $K=G_{1} \cap G_{2}$ is a complete graph with $|V(K)|=$ $k$, then $G=G_{1} \cup G_{2}$ is called the k-clique-sum (or clique-sum) of $G_{1}$ and $G_{2}$ in $K$.

Corollary 50. If $G$ is the 2-clique-sum of the cycles $C_{1}$ and $C_{2}$ with $\left|V\left(C_{1}\right)\right|=r_{1} \leqslant r_{2}=$ $\left|V\left(C_{2}\right)\right|$, then $G$ is vertex decomposable if and only if $r_{1}=3$ or $r_{1}=r_{2}=5$.

Proof. $\Leftarrow)$ First, we suppose that $r_{1}=3$. Consequently, we can assume $C_{1}=\left(x_{1}, x_{2}, x_{3}\right)$ and $x_{2}, x_{3} \in V\left(C_{1}\right) \cap V\left(C_{2}\right)$. Thus, $x_{1}$ is a simplicial vertex. Hence, by Remark $7, x_{2}$ is a shedding vertex. Furthermore, $G \backslash x_{2}$ and $G \backslash N_{G}\left[x_{2}\right]$ are trees. Consequently, by Remark 31, $G \backslash x_{2}$ and $G \backslash N_{G}\left[x_{2}\right]$ are vertex decomposable graphs. Therefore, $G$ is vertex decomposable.

Now, we assume that $r_{1}=r_{2}=5$ with $C_{1}=\left(x_{1}, x_{2}, x_{3}, x_{4}, x_{5}\right)$ and $C_{2}=\left(y_{1}, x_{2}, x_{3}, y_{4}\right.$, $\left.y_{5}\right)$. We take a stable set $S$ in $G \backslash N_{G}\left[x_{5}\right]$. If $x_{2} \in S$, then $S \cup\left\{x_{4}\right\}$ is a stable set in $G_{1}=G \backslash x_{5}$. If $x_{2} \notin S$, then $S \cup\left\{x_{1}\right\}$ is a stable set in $G_{1}$. Consequently, by Lemma $2, x_{5}$ is a shedding vertex. Since $x_{2}$ is a neighbor of a free vertex in $G_{1}$, then $x_{2}$ is a shedding vertex in $G_{1}$. Furthermore, since $G_{1} \backslash x_{2}$ and $G_{1} \backslash N_{G_{1}}\left[x_{2}\right]$ are forests, then they are vertex decomposable graphs, by Remark 31. Thus, $G_{1}$ is vertex decomposable. Snce $G \backslash N_{G}\left[x_{5}\right]=C_{2}$, it is vertex decomposable by Remark 31 . Therefore, $G$ is vertex decomposable.

$\Rightarrow)$ By Corollary 47, we have that $\left\{r_{1}, r_{2}\right\} \cap\{3,5\} \neq \varnothing$. We suppose $r_{1} \neq 3$. So $r_{1}=5$ or $r_{2}=5$. Consequently, we can assume that $\left\{C_{1}, C_{2}\right\}=\left\{C, C^{\prime}\right\}$ where $C=\left(x_{1}, x_{2}, x_{3}, x_{4}, x_{5}\right)$ and $x_{2}, x_{3} \in V(C) \cap V\left(C^{\prime}\right)$. Thus, $G \backslash N_{G}\left[x_{5}\right]=C^{\prime}$ is vertex decomposable. Hence, from Remark 31, $\left|V\left(C^{\prime}\right)\right| \in\{3,5\}$. But $r_{1} \neq 3$, then $\left|V\left(C^{\prime}\right)\right|=5$ and $r_{1}=r_{2}=5$. Therefore, $r_{1}=3$ or $r_{1}=r_{2}=5$.

Lemma 51. Let $G$ be a 2-connected graph with girth at least 11. Then $G$ is not shellable.

Proof. Since $G$ is 2-connected, then $G$ is not a forest. Consequently, if $r$ is the girth of $G$, then there exists a cycle $C=\left(x_{1}, x_{2}, \ldots, x_{r}\right)$. If $G=C$, then $G$ is not shellable. Hence, $G \neq C$ implying $D_{1}(C) \neq \varnothing$. We take $y \in D_{1}(C)$, without loss of generality we can assume that $\left\{x_{1}, y\right\} \in E(G)$. If $\left\{x_{i}, y\right\} \in E(G)$ for some $i \in\{2, \ldots, r\}$, then we take the cycles $C_{1}=\left(y, x_{1}, x_{2}, \ldots, x_{i}\right)$ and $C_{2}=\left(y, x_{1}, x_{r}, x_{r-1}, \ldots, x_{i}\right)$. Thus, $\left|V\left(C_{1}\right)\right|=i+1$ and $\left|V\left(C_{2}\right)\right|=r-i+3$. Since $r$ is the girth of $G$, then $i+1 \geqslant r$ and $r-i+3 \geqslant r$. Consequently, $3 \geqslant i$ implies $4 \geqslant r$. But $r \geqslant 11$, this is a contradiction. This implies that $\left|N_{G}(y) \cap V(C)\right|=1$. Now, we suppose that there exist $y_{1}, y_{2} \in D_{1}(C)$ such that $\left\{y_{1}, y_{2}\right\} \in$ $E(G)$. We can assume that $\left\{x_{1}, y_{1}\right\},\left\{x_{i}, y_{2}\right\} \in E(G)$. Since $r \geqslant 11$, then there are no 3 -cycles in $G$. In particular, $x_{1} \neq x_{i}$. Now, we take the cycles $C^{\prime}=\left(y_{1}, x_{1}, \ldots, x_{i}, y_{2}\right)$ and $C^{\prime \prime}=\left(y_{1}, x_{1}, x_{r}, x_{r-1}, \ldots, x_{i}, y_{2}\right)$. So, $\left|V\left(C^{\prime}\right)\right|=i+2$ and $\left|V\left(C^{\prime \prime}\right)\right|=r-i+4$. Since $r$ is the girth, we have that $i+2 \geqslant r$ and $r-i+4 \geqslant r$. Hence, $4 \geqslant i$ and 
$6 \geqslant r$, this is a contradiction. Then $D_{1}(C)$ is a stable set. Now, since $G$ is 2-connected, then for each $y \in D_{1}(C)$ there exists $z \in N_{G}(y) \cap D_{2}(C)$. If there exist $z_{1}, z_{2} \in D_{2}(C)$ such that $\left\{z_{1}, z_{2}\right\} \in E(G)$, then there exist $y_{1}, y_{j} \in D_{1}(C)$ such that $\left\{z_{1}, y_{1}\right\},\left\{z_{2}, y_{j}\right\} \in$ $E(G)$. Since there are no 3-cycles in $G$, we have that $y_{1} \neq y_{j}$. We can assume that $\left\{x_{1}, y_{1}\right\},\left\{x_{i}, y_{j}\right\} \in E(G)$. Since there are no 5 -cycles, then $i \neq 1$. Consequently, there exist cycles $C_{1}^{\prime}=\left(x_{1}, \ldots, x_{i}, y_{j}, z_{2}, z_{1}, y_{1}\right)$ and $C_{2}^{\prime}=\left(x_{i}, \ldots, x_{r}, x_{1}, y_{1}, z_{1}, z_{2}, y_{j}\right)$. This implies $r \leqslant\left|V\left(C_{1}^{\prime}\right)\right|=i+4$ and $r \leqslant\left|V\left(C_{2}^{\prime}\right)\right|=r-i+6$. Hence, $i \leqslant 6$ and $r \leqslant 10$, this is a contradiction. Then $D_{2}(C)$ is a stable set. Furthermore, $C$ is a connected component of $G \backslash N_{G}\left[D_{2}(C)\right]$. But $C$ is not shellable, therefore $G$ is not shellable, from Remark 26.

Theorem 52. If $G$ has girth at least 11, then $G$ is shellable if and only if there exists $x \in V(G)$ with $N_{G}(x)=\{y\}$ such that $G \backslash N_{G}[x]$ and $G \backslash N_{G}[y]$ are shellable.

Proof. $\Leftarrow)$ By $([18]$, Theorem 2.9).

$\Rightarrow$ ) By Remark 26, shellability is closed under c-minors. Consequently, it is only necessary to prove that $G$ has a free vertex. If every block of $G$ is an edge or a vertex, then $G$ is a forest and there exists $x \in V(G)$ with $\operatorname{deg}_{G}(x)=1$. Hence, we can assume that there exists a 2-connected block $B$ of $G$. The girth of $B$ is at least 11 , since $B$ is an induced subgraph of $G$. Thus, by Lemma $51, B$ is not shellable. Therefore, by Theorem 42 , there exists $x \in D_{1}(B)$ such that $\operatorname{deg}_{G}(x)=1$.

\section{Acknowledgements}

The authors are grateful to the referees whose suggestions improved the presentation of this paper.

\section{References}

[1] J. Biermann, C. A. Francisco, and H. Tài Hà, A. Van Tuyl. Partial coloring, vertex decomposability and sequentially Cohen-Macaulay simplicial complexes. J. Commut. Algebra, 7(3):337-352, 2015.

[2] T. Biyikoğlu and Y. Civan. Vertex-decomposable graphs, codismantlability, CohenMacaulayness, and Castelnuovo-Mumford regularity. Electron. J. Combin., 21(1) 2014, \#P1.1.

[3] A. Bruns and J. Herzog. Cohen-Macaulay Ring. Cambridge University Press, Cambridge, 1998.

[4] I. D. Castrillón and R. Cruz. Escalonabilidad de grafos e hipergrafos simples que contienen vértices simpliciales. Matemáticas: Enseñanza Universitaria, XX(1):6980, 2012.

[5] M. Crupi, G. Rinaldo, and N. Terai. Cohen-Macaulay edge ideal whose height is half of the number of vertices. Nagoya Math. J., 201:117-131, 2011.

[6] A. Dochtermann and A. Engström. Algebraic properties of edge ideals via combinatorial topology. Electron. J. Combin., 16(2), 2009. 
[7] M. Estrada and R. H. Villarreal. Cohen-Macaulay bipartite graphs. Arch. Math., 68(2):124-128, 1997.

[8] A. Finbow, B. Hartnell,and R. J. Nowakowski. A characterization of well covered graphs of girth 5 or greater. J. Combin. Theory Ser. B, 57(1):44-68, 1993.

[9] C. A. Francisco and A. Van Tuyl. Sequentially Cohen-Macaulay edge ideals. Proc. Amer. Math. Soc. (electronic), 135(8):2327-2337, 2007.

[10] I. Gitler and C. E. Valencia. On bounds for some graph invariants. Bol. Soc. Mat. Mexicana, 16(2):73-94, 2010.

[11] J. Herzog and T. Hibi. Distributive lattices, bipartite graphs and Alexander duality. J. Algebraic Combin., 22:289-302, 2005.

[12] D. T. Hoang, N. C. Minh, T. N Trung. Cohen-Macaulay graphs with large girth. J. Algebra Appl., 14(7), 2015.

[13] M. Mahmoudi, A. Mousivand, M. Crupi, G. Rinaldo, N. Terai, and S. Yassemi. Vertex decomposability and regularity of very well-covered graphs. J. Pure Appl. Algebra, 215(10):2473-2480, 2011.

[14] S. Morey, E. Reyes, and R. H. Villarreal. Cohen-Macaulay, shellable and unmixed clutters with perfect matching of König type. J. Pure Appl. Algebra, 212(7):17701786, 2008.

[15] G. Ravindra. Well-covered graphs. J. Combin. Inform. System Sci. 2(1):20-21, 1977.

[16] R. P. Stanley. Combinatorics and Commutative Algebra. Second edition, Progress in Mathematics 41. Birkhäuser Boston, Inc., Boston, MA. 1996.

[17] A. Van Tuyl. Sequentially Cohen-Macaulay bipartite graphs: vertex decomposability and regularity. Arch. Math., 93:451-459, 2009.

[18] A. Van Tuyl, and R. H. Villarreal. Shellable graphs and sequentially Cohen-Macaulay bipartite graphs. J. Combin. Theory Ser. A, 115(5):799-814, 2008.

[19] R. H. Villarreal. Unmixed bipartite graphs. Rev. Colombiana Mat., 41(2):393-395, 2007.

[20] R. H. Villarreal. Monomial Algebras. Second edition, Monographs and Research Notes in Mathematics, Chapman \& Hall/CRC. 2015.

[21] M. L Wachs. Obstructions to shellability. Discrete Comput. Geom., 22(1):95-103, 1999.

[22] R. Woodroofe. Vertex decomposable graphs and obstructions to shellability. Proc. Amer. Math. Soc., 137:3235-3246, 2009. 